Diktaturen und die emeute Hinwendung zu gewählten Präsidenten zeige, daß von einem regelrechten Zyklus gesprochen werden könne.

Die zeitgenössischen Ereignisse in Südamerika scheinen diese Interpretation, die von verschiedenen Politikwissenschaftlicher geteilt wird, zu bestätigen. Nach der Krise der Diktaturen gelangten in fast allen südamerikanischen Staaten liberal-demokratische Präsidenten an die Regierung. Sie betonten die Notwendigkeit parlamentarisch-präsidentieller Demokratie, unterschieden sich in der Wirtschaftspolitik allerdings kaum von dem neoliberalen Kurs der Militärs. Die hohen Erwartungen der Bevölkerung und der Intellektuellen in die "neuen Demokratien" begannen bald einer Emüchterung zu weichen. Die strafrechtliche Verfolgung der Menschenrechtsverletzungen wurde, sofern sie überhaupt begonnen hatte, unter dem Druck der Generäle eingestellt, die Verschuldung sowie der Schuldendienst stiegen weiter an, und von einigen Präsidenten begannen Fälle von Nepotismus und Mißwirtschaft bekannt zu werden, die schon in den 60er Jahren zur Diskreditierung der Demokratie in Lateinamerika beigetragen hatten. Gegenüber dem universalistischen Diskurs der amtierenden Präsidenten, von denen sich besonders Rául Alfonsín in Argentinen stark an Europa orientierte, erleben populistisch-nationalistische Politiker eine Renaissance. In Venezuela, Argentinien und Brasilien haben sie gute Chancen, die kommenden Wahlen zu gewinnen. Peru kann mit der Ablösung des konservativ-liberalen Belaunde Terry durch den zunächst populären Apristen Alan Garcia als Vorreiter gelten.

\title{
Demokratie und lateinamerikanische Gesellschaftsstrukturen
}

\author{
Von Wolfgang Spoerr
}

Manfred Mols, Demokratie in Lateinamerika, Stuttgart, Berlin, Köln, Mainz, Urban Taschenbücher, Kohlhammer, 1985, 220 S., DM 28,-

Wolfgang Dietrich, Honduras - ein Demokratisierungsversuch zwischen Repression, Revolution und Resignation, Reihe Heidelberger Dritte Welt Studien, Bd. 22, Heidelberger Verlagsanstalt und Druckerei, 1987, 321 S., DM 29,-

Ausgehend von der normativen Vorgabe des Konzeptes einer "offenen, pluralistischen Demokratie" westlichen Musters analysiert der Mainzer Politikwissenschaftler Manfred Mols deren Verwirklichungs- und Stabilisierungschancen. Konsequent für eine Studie, die 
die iberoamerikanischen Staaten zwischen Rio Grande und Ushuaia gemeinsam und länderübergreifend abhandelt, bilden die "Legate einer belastenden Tradition" das erste Thema, das Mols detailliert erörtert. Die spanische Kolonialzeit zeichne sich durch einen Dualismus zwischen (seinerzeit) moderner autoritär-bürokratisch-zentralistischer Staatsorganisation und feudaler Gesellschaftsstruktur aus. Dieser innere Widerspruch, verbunden mit dem hohen Modernisierungs- und Adaptionsdruck, der auf lateinamerikanischen Staaten lastete und lastet, wirke bis heute fort. Der Versuch, das Staatswesen zu modemiesieren, ohne die gesellschaftlichen Strukturen anzupassen, sei bereits im 19. Jahrhundert gescheitert.

Bleibende Resultate dieser historischen Gegebenheiten seien heute die inneren Widersprüche Lateinamerikas, die Mols u.a. anhand der Begriffspaare Caudillismus / TechnokratenHerrschaft, Prätorianismus / Populistischer Korporatismus, Zivilismus / Militarismus und urbane/ländliche Kultur festmacht. Bei der Erörterung dieser Phänomene wird die Nähe des Verfassers zum "political-culture"-Ansatz deutlich, der im Unterschied zu institutionelltypologischen Analysen stark auf systemübergreifend vorhandene Verhaltensmuster politischer Akteure abhebt.

Aufgrund der unbefriedigenden gegenwärtigen Situation stellt sich die Frage nach dem Demokratiepotential der politischen Kräfte und Institutionen, vor allem als Frage nach ihrem Wandlungspotential. Die allgemeine Skepsis gegenüber der Partizipationsfähigkeit und -bereitschaft der Unterschichten wird von Mols relativiert. Auch die Einschätzung der Rolle der Mittelschichten, denen nach berechtigter Auffassung vieler Autoren eine Schlüsselrolle als Demokratieträger zukommt, fällt differenziert aus. Der Verweis auf die zwielichtige Rolle der Mittelschichten im gegenwärtigen Mexiko der Institutionalisierten Revolution und in Brasilien zur Zeit des Militärputsches (1964) freilich unterstreicht die Schlüsselrolle der Mittelschichten für die Stabilisierung pluralistischer Demokratie eher noch.

Wenig positiv fällt das Urteil über das Wandlungspotential der Untemehmerschaft aus. Eine wesentlich geringere Rolle in der nationalen Politik spiele zwar heute der traditionelle Teil der Agrarbourgeoisie, die "latifundistas", ihr Wandlungspotential sei aber gering einzuschätzen. Gewichtiger erscheint insbesondere, daß sie - worauf Mols zurecht hinweist Träger der pseudofeudalen "patrón / peón"-Verhältnisse sind, die die politische Kultur auf dem Lande prägen.

Am lateinamerikanischen Gewerkschaftswesen kritisiert der Verfasser inteme Mißstände "Bossismus" und Kazikentum - und gelegentliche politische Willfährigkeit gegenüber undemokratischen Kräften. Hinzufügen sollte man, daß sie die Gewerkschaften Lateinamerikas vielerorts als Hüter der Privilegien einer kleinen Schicht von Industriearbeitern gebären - um den Preis hoher gesamtwirtschaftlicher Nachteile und ohne daß die wirklich Benachteiligten (Campesinos, Marginalisierte) davon profitieren. Positiv schätzt der Autor die (neue) Rolle der katholischen Kirche ein.

Einer eingehenden Untersuchung wird schließlich die lateinamerikanische Bürokratie unterzogen. Hier kommmt der Autor für die gemeinhin als aufgebläht, korrupt und ineffi- 
zient geltenden Staatsapparate zu einer Neubewertung. Großen Modemisierungsanstrengungn und auch -leistungen ständen eine mangelhafte Ressourcenausstattung (in Bolivien habe ein Minister 198440 US\$ monatliches Gehalt erhalten), geringe Institutionalisierung (Personalismus, Klientelismus und Beutesystem) und eine teilweise überzogene und wirtschaftlich nicht verantwortbare Sozialgesetzgebung gegenüber. Die Betrachtung der Rolle der Gerichte führt den Autor zu dem Ergebnis, in Lateinamerika könne "im allgemeinen nicht von Rechtsstaatlichkeit" geredet werden (S. 160), eine Formulierung, die überzogen und undifferenziert erscheint.

Obwohl sich, mit Ausnahme Kubas und Nicaraguas, alle Staaten Lateinamerikas zumindest verbal zur okzidental-pluralistischen Demokratie bekennen und auch in den lateinamerikanischen Sozialwissenschaften sich ein breiterer Konsens darüber abzeichne, bleibt Mols im Ergebnis skeptisch. Seine Hoffnungen ruhen auf den demokratischen Reformparteien, auf der ebenfalls geringen Stabilität autoritärer Systeme und auf nur ansatzweise gestreiften institutionellen Reformen (Parlamentarismus statt Präsidentialismus, Abschaffung des Wiederwahlverbotes für Parlamentarier). Ob freilich die Stärkung der "Verhandlungsmacht der beteiligten und/oder betroffenen Gruppen und Verbände" hilfreich ist, mag mit Blick auf die argentinischen Erfahrungen bezweifelt werden. Wichtiger erscheine eine Verbreiterung der organisierten Interessen, ein erhöhtes gesamtwirtschaftliches Verantwortungsbewußtsein und mehr Demokratiebewußtsein im Bereich der Interessenvertretungen.

Nicht zuletzt durch die umfassende, aber doch nie weitschweifige Berücksichtigung auch der nord- und südamerikanischen Literatur ist Mols eine äußerst lesenswerte Darstellung lateinamerikanischer Gegenwart gelungen. Die Konzentration auf Aspekte der politischen Kultur ist eine wertvolle Ergänzung der sonst vorherrschenden, modernisierungstheoretischen und Dependencia-Ansätze. Sie hält der Autor für stillschweigende Partner einer "unheiligen Allianz" (S. 70), die sich allesamt als wenig hilfreich für die Bewältigung des belastenden Problemdrucks erwiesen hätten. An für eine länderübergreifende Studie unvermeidliche Grenzen stößt der Arbeit vor allem da, wo länderspezifische Besonderheiten zur Vervollständigung und Ausdifferenzierung des Bildes unersetzlich sind. Die immer wieder vorkommenden Länderbeispiele können dieses Defizit nicht in vollem Umfange ausgleichen.

Wie wertvoll landesspezifische Besonderheiten und historische Details zur Vervollständigung des Lateinamerika-Bildes sind, zeigt die Studie von Wolfgang Dietrich. Sie schließt eine Lücke, denn bisher gab es zu diesem zentralamerikanischen Land nur wenig Literatur in deutscher Sprache. Dietrich erklärt, wie es zu der im Vergleich zu den Nachbarländern El Salvador und Nicaragua völlig unterschiedlichen Situation kam. Während in den Nachbarländern am Ende der siebziger Jahre eine sozialrevolutionäre Situation bestand, blieb diese in Honduras aus. Die fehlende historische Tradition antiimperialistischen und nationalen Befreiungsdenkens, das Fehlen einer Krise innerhalb der Mittelschichten, geringere Vermögensgegensätze, kein langjähriger Diktator als verhaßter "Buhmann" des Volks- 
zomes, das geringe Bildungsniveau und die damit einhergehende Apathie weiter Teile der Bevölkerung seien die Ursachen.

Die Zeit nach der kurz dargestellten spanischen Kolonialherrschaft erörtert der Verfasser periodisiert, wobei den Abschnitten jeweils ein eigenes Kapitel zur US-amerikanischen Außenpolitik zur Seite gestellt wird. Ob freilich die Monroe-Doktrin allein als Ergebnis rassistischer in Verbindung mit imperialistischen Uberlegungen erklärt werden kann, scheint zweifelhaft. Schließlich waren die Vereinigten Staaten zu dieser Zeit selbst noch ein Staat, dessen innere Situation keinesfalls konsolidiert war. Die Monroe-Doktrin war zumindest auch gemeinsame Abwehr gegen die seinerzeitigen Großmächte Europas. Die von Dietrich vorgenommene Interpretation der amerikanischen Geschichte erscheint insgesamt zu sehr von heutiger Sicht geprägt.

Dietrich beschreibt weiter, wie sich in den letzten drei Jahrzehnten des 19. Jahrhunderts das entwickelte, was heute als "Bananenrepublik" in die Umgangssprache eingegangen ist. Im Austausch gegen verkehrsmäßige Erschließung erhielten die nordamerikanischen Fruchtgesellschaften riesige Ländereien in den vorher unerschlossenen und dünn besiedelten atlantischen Küstengebieten. Dies führte schließlich dazu, daß die politischen Systeme der betroffenen Staaten ihre Autonomie weitgehend einbüßten. Die Folgen für Zentralamerika waren verheerend - dies mag auch sprachliche Entgleisungen Dietrichs in den betreffenden Kapiteln erklären. So schreibt er, die amerikanischen Regierungen seit dem ersten Weltkrieg seien die "politischen Kretins" der "Wirtschaftsgiganten" gewesen (S. 79). Die Unterstützung der hondurenischen Regierung für die guatemaltekischen Putschisten 1954 bezeichnet Dietrich als "Kadavergehorsam gegenüber den nordamerikanischen Befehlsgebem" und "besonders ekelhaftes Beispiel für die Charakterlosigkeit und Käuflichkeit hondurenischer Politiker" (S. 87).

Je näher der Autor der Gegenwart kommt, desto abschweifender werden die jeweiligen Ausführungen zu den USA. So wird seitenlang der kubanische Fall erörtert und dabei der Weg Castros in den Sozialismus marxistisch-leninistischer Prägung und die totale Abhängigkeit von der Sowjetunion historisch unhaltbar ausschließlich als Folge der US-amerikanischen Politik erklärt (S. 136-141).

Undifferenziert behandelt der Autor auch die multinationalen Unternehmen. Mit Allgemeinheitsanspruch für ganz Lateinamerika erklärt er, daß die "Gewinnmaximierung der Multis eben" impliziere, daß man die Rohstoffpreise "bis an die Grenze des Diebstahls und darunter" drücke. So schlage "bildlich gesprochen in den Adern der nordamerikanischen Wirtschaft zentralamerikanisches und anderes Blut und diese laufende Transfusion bewirkt, daß der eine Körper immer stärker und größer wird, während die ausgebluteten Kreisläufe krank und schwach werden". Wie hoch die Gewinnraten der "Multis" wirklich liegen, schreibt Dietrich nicht. Daß viele, wenn nicht sogar die Mehrzahl nicht im Rohstoffhandel, sonderm in der Produktion tätig sind, bleibt ebenfalls außer Betracht. Daß bedeutende Industrialisierungsleistungen gerade zusammen mit den transnationalen Unternehmen erfolgten (beispielsweise in Brasilien), berichtet Dietrich ebenfalls nicht. 
Mit der Amtsübernahme Ronald Reagans verliert Dietrich sein Thema noch mehr aus dem Auge: Fast eine Seite lang wird der "SDI Rüstungswahn" abgehandelt (S. 244); nicht nur unhistorisch, sondern auch geschmacklos die Aussagen auf S. 242, der "von Reagan herbeigeführte Aufschwung erfolgte nach einenm ähnlichen Prinzip wie der Hitler'sche". Unter Berufung auf Joan Baez wird der amerikanische Präsident denn auch als "Head of the Klukluxclan" (S. 240) bezeichnet und schließlich seine Politik als "schlicht faschistisch" diffamiert (S. 242). Auch bei der Erörterung der innerhondurenischen Situation bleibt derartiges leider nicht aus: Der Leser erfährt beispielsweise, daß amerikanische Soldaten AIDS und Geschlechtskrankheiten einschleppten und daß die amerikanischen Truppen in Manövern "Yellow Rain und andere chemische Kampfstoffe" einsetzten (S. 181).

Eine Menge interessanter Details fördern dagegen die Kapitel zur aktuellen inneren Situation im seit 1982 demokratischen (Dietrich: "formaldemokratischen") Honduras zu Tage, insbesondere über die staatliche Bürokratie, das Militär, die Parteien und Interessenvertretungen sowie die Kirche. Wo Dietrich auf die Situation in den Nachbarländern eingeht, wird er dieser nicht immer gerecht. So beispielsweise, wenn er der nicaraguanischen, antimarxistischen Guerilla vorhält, sie betreibe "Massaker gegen die Zivilbevölkerung, Sabotageakte gegen Wirtschaftseinrichtungen und fiese Hinterhalte gegen militärische (!) und zivile Transporte" (S. 185), andererseits aber die marxistische salvadorenische Guerilla stets romantisch-verklärt beschreibt. Dabei hat gerade sie das ehemalige "Japan Mittelamerikas" durch ihre gezielten Sabotageakte gegen Wirtschaftseinrichtungen um Jahrzehnte zurückgeworfen. In der Osthälfte des Landes bleibt kaum eine Brücke von den Sprengladungen der Guerillaorganisation FMLN verschont. Dies und die Verminung landwirtschaftlicher Produktions- und anderer Flächen, die schuld ist an der Verkrüppelung von Hunderten Frauen und Kindem, erwähnt Dietrich nicht. Ärgerlich ist es auch, daß Dietrich die Opfer der rechtsradikalen salvadorenischen "Todesschwadronen", die die christdemokratische, gewählte Regierung Präsident Duartes (oft in stillschweigender Allianz mit der linksgerichteten Guerilla) bekämpfen, pauschal der Regierung zurechnet (S. 250). Auch wenn die Verbindung von Teilen der Sicherheitskräfte mit den Mordkommandos nicht zu bestreiten ist, werden derartige undifferenzierte Statistiken der lateinamerikanischen Realität und der sie kennzeichnenden Spannung zwischen rechtsgerichteten Kreisen im Militär und demokratisch gewählten Reformregierungen nicht gerecht. 